\title{
Ultrasonic-Assisted Extraction, Structural Characterization, Chain Conformation, and Biological Activities of a Pectic-Polysaccharide from Okra (Abelmoschus esculentus)
}

\author{
Xi-Rui Nie ${ }^{\dagger}$, Yuan $\mathrm{Fu}^{\dagger}{ }^{\dagger}$, Ding-Tao Wu *(D), Ting-Ting Huang, Qin Jiang, Li Zhao, Qing Zhang ${ }^{\circledR}$, \\ De-Rong Lin, Hong Chen ${ }^{(D)}$ and Wen Qin * \\ College of Food Science, Sichuan Agricultural University, Ya'an 625014, China; niexrachel@163.com (X.-R.N.); \\ yuanffuy@163.com (Y.F.); HTT84640527@163.com (T.-T.H.); xinchenggui@163.com (Q.J.); \\ zhaoli0608@126.com (L.Z.); zhangqing@sicau.edu.cn (Q.Z.); lindr2018@sicau.edu.cn (D.-R.L.); \\ chenhong945@sicau.edu.cn (H.C.) \\ * Correspondence: DT_Wu@sicau.edu.cn (D.-T.W.); Qinwen@sicau.edu.cn (W.Q.); \\ Tel.: +86-0835-2883219 (D.-T.W.); Fax: +86-0835-2883219 (D.-T.W.) \\ + These authors contributed equally to this work.
}

Received: 22 February 2020; Accepted: 3 March 2020; Published: 5 March 2020

check for updates

\begin{abstract}
The purpose of this study was to better understand the chemical characteristics and chain conformation of okra polysaccharides extracted by ultrasonic-assisted extraction. A pectic-polysaccharide, named OPP-D, was obtained, which was mainly composed of rhamnose, galacturonic acid, and galactose with a molar ratio of 1.01:1.00:2.31. Combined with NMR analysis, -4$)-\alpha$-D-GalA $p$-(1,2,4)- $\alpha$-L-Rhap-(1- were identified as the backbone with galactan side chains substituted partly at O-4 of Rhap. Molecular weight and radius of gyration of OPP-D were determined as $2.19 \times 10^{5} \mathrm{Da}$ and $27.0 \mathrm{~nm}$, respectively. OPP-D was determined as an air-core sphere with branching chains in $0.9 \% \mathrm{NaCl}$ solution by high-performance size-exclusion chromatography coupled with multi-angle laser light scattering and dynamic light scattering for the first time. Moreover, OPP-D exhibited typical shear-thinning behavior. In addition, OPP-D exhibited remarkable in vitro antioxidant activities and prebiotic activities, while the relatively high molecular weight, high degree of esterification, high content of uronic acids, and highly branched globular conformation of OPP-D might contribute to its in vitro anti-diabetic activities and binding capacities. Results can contribute to a better understanding of the structure-bioactivity relationship of OPPs, and OPP-D has great potential applications in the functional food and pharmaceutical industries.
\end{abstract}

Keywords: okra polysaccharides; structural characteristics; chain conformation; biological activities

\section{Introduction}

Abelmoschus esculentus (L.) Moench, a delicious vegetable known as okra, has been used worldwide as a functional food and folk medicine for many years [1]. It is native to Africa and has been cultivated in China [2,3]. Previous studies have reported that okra has abundant bioactivities, including anti-fatigue [2], antioxidant [4], anti-hyperglycemic, and anti-diabetic effects [5]. Generally, one of the major health-promoting properties in okra is referred to as polysaccharides [6]. Okra polysaccharides (OPPs) extracted by hot water extraction have been investigated. Generally, OPPs have been reported to possess relatively high molecular weights ranging from $2.99 \times 10^{6}$ to $5.94 \times 10^{6} \mathrm{Da}[7,8]$. The relatively high content of uronic acids in OPPs confirms the presence of pectic-polysaccharides [9]. Moreover, the major constituent monosaccharides of OPPs are determined as rhamnose, galacturonic acid, galactose, 
and arabinose [10]. Nevertheless, the chain conformation of OPPs and their structure-bioactivity relationships are still insufficient.

Generally, extraction methods have noticeable influences on the physicochemical characteristics and bioactivities of OPPs [11]. Ultrasonic-assisted extraction (UAE), as a green extraction method, has already been widely exploited for accelerating the extraction processes of polysaccharides [12]. UAE is utilized due to the acoustic cavitation to destroy cell walls, reduce particle sizes, and enhance the contact between solvents and targeted compounds $[13,14]$. UAE has several advantages, such as short extraction time, moderate solvent requirements, and minimal impacts on bioactivities [4]. Previous studies have investigated the extraction optimization and antioxidant activities of OPPs extracted by UAE, which confirm that the UAE method can be an efficient method to extract OPPs with high antioxidant activities [4]. However, the detail chemical structures and chain conformation of OPPs extracted by UAE, as well as their structure-bioactivity relationships are still unknown.

Therefore, in order to well understand the detail chemical structures and chain conformation of OPPs and improve the applications of OPPs in the functional food and pharmaceutical industries, the purification, structural characteristics, chain conformation, and bioactivities of OPPs extracted by UAE were investigated.

\section{Results and Discussion}

\subsection{Physicochemical Characteristics}

\subsubsection{Chemical Compositions of OPP-D}

The extraction yield of OPPs extracted by UAE was $13.99 \% \pm 0.52 \%$, which was comparable to the extraction yields of OPPs extracted by microwave-assisted extraction (MAE), hot water extraction (HWE), and pressurized water extraction (PWE) previously reported [11]. The extraction time of UAE was much shorter than those of HWE and PWE, and the extraction temperature of UAE was also lower than those of HWE, PWE, and MAE, which suggested that the UAE method was a more efficient and green method.

Moreover, the chemical compositions of the purified OPP (OPP-D) were determined. The content of total polysaccharides in OPP-D was determined to be $92.85 \% \pm 2.27 \%$, and the content of proteins was $0.33 \% \pm 0.07 \%$, which suggested that polysaccharides were the main biological components in OPP-D. Moreover, the content of uronic acids in OPP-D was determined as $33.35 \% \pm 2.38 \%$. In general, such high content of uronic acids in natural polysaccharides might lead to strong antioxidant activities, digestive enzyme inhibitions, and binding properties $[9,15,16]$. However, the content of total uronic acids in OPP-D extracted by UAE was significantly lower than that of OPPs extracted by MAE, HWE, and PWE [11], which might be attributed to the degradation of homogalacturonan (HG) in OPP under the ultrasonic condition $[14,17]$.

\subsubsection{Constituent Monosaccharides of OPP-D}

Monosaccharides are the natural basic units that determine the unique structures and properties of polysaccharides [11]. The monosaccharide composition of OPP-D was investigated on the basis of PMP pre-column derivation by high-performance liquid chromatography (HPLC). As shown in Figure 1, the major constituent monosaccharides of OPP-D were measured as rhamnose (Rha), galacturonic acid (GalA), and galactose (Gal). The molar ratio of Rha, GalA, and Gal in OPP-D was determined to be about 1.01:1.00:2.31, respectively. This demonstrated that OPP-D was a pectic-polysaccharide. In addition, the close molar ratio of Rha and GalA indicated that they might be the residues building the backbone, while galactose might be the residue constructing the side chains as it had the highest molar ratio $[10,18]$. 


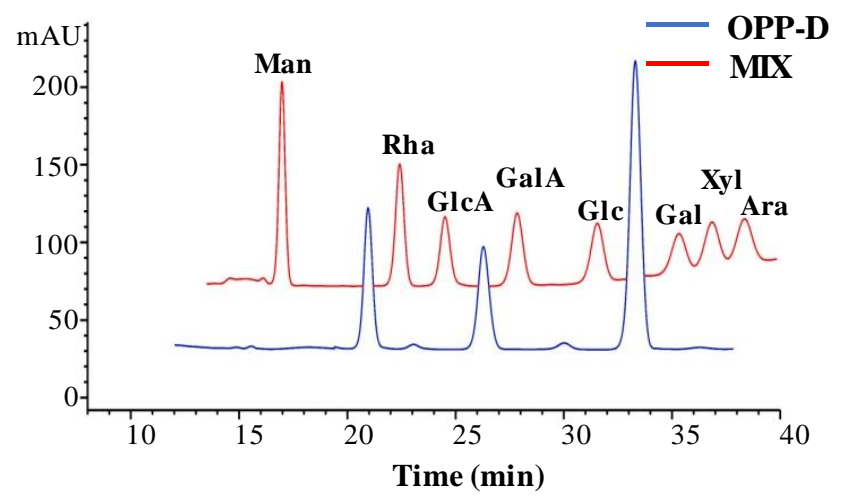

Figure 1. High-performance liquid chromatography profile of purified okra polysaccharides (OPP-D). Man, mannose; Rha, rhamnose; GlcA, glucuronic acid; GalA, galacturonic acid; Glc, glucose; Gal, galactose; Xyl, xylose; Ara, arabinose.

\subsubsection{FT-IR Spectra and Esterification Degree of OPP-D}

In order to determine the structural features of OPP-D, the FT-IR spectra were measured. As shown in Figure 2, the broad band at $3262.62 \mathrm{~cm}^{-1}$ corresponds to the hydroxyl group stretching vibration [19]. The absorption peak at $2931.39 \mathrm{~cm}^{-1}$ is attributed to the stretching vibration of C-H asymmetric [19]. Then, the absorption peaks at $1724.12 \mathrm{~cm}^{-1}$ and $1628.48 \mathrm{~cm}^{-1}$ are owing to esterified groups of the $\mathrm{C}=\mathrm{O}$ and $\mathrm{COO}$ - stretching vibration, which indicates the presence of uronic acids [11]. Results were consistent with the constituent monosaccharides of OPP-D. The absorption peaks at $1417.00 \mathrm{~cm}^{-1}$ and $1147.97 \mathrm{~cm}^{-1}$ are owing to the $\mathrm{C}-\mathrm{H}$ or $\mathrm{O}-\mathrm{H}$ and $\mathrm{C}-\mathrm{O}-\mathrm{C}$ bending vibration, which indicates the existence of -OCH3 [9]. Furthermore, the degree of esterification (DE) of OPP-D was calculated as $22.81 \% \pm 0.85 \%$. Previous studies have reported that the high DE in natural polysaccharides may lead to high inhibition of digestive enzymes [20], while the low DE may lead to high antioxidant activities $[19,21]$. Furthermore, results suggested that the chemical structure of OPP-D was similar to the chemical structures of OPPs extracted by MAE, HWE, and PWE, but the DE in OPP-D was lower than the DE in OPP extracted by HWE and PWE [11]. This might be related to the degradation of OPP-D under the ultrasonic condition [17].

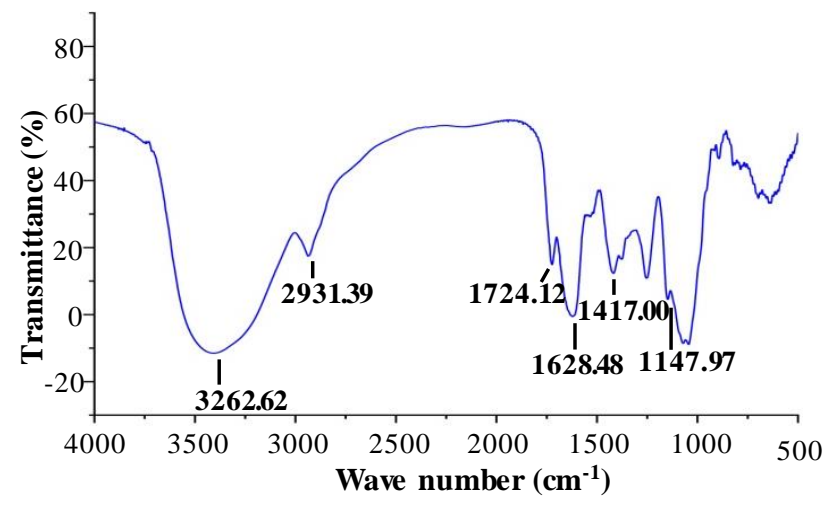

Figure 2. FT-IR spectra of OPP-D.

\subsubsection{Structure Prediction of OPP-D by NMR Analysis}

NMR spectra were recorded in order to reveal the precise structural information of OPP-D. 1D NMR spectra, including ${ }^{1} \mathrm{H}$ and ${ }^{13} \mathrm{C}$ analyses, were shown in Figure $3 \mathrm{~A}, \mathrm{~B}$. The ${ }^{1} \mathrm{H}$ signal at $4.80 \mathrm{ppm}$ belongs to $\mathrm{D}_{2} \mathrm{O}$. In combination with the constituent monosaccharides of OPP-D, the abroad peaks at 1.26 and $1.35 \mathrm{ppm}$ are attributed to the H-6 of $1,2-\alpha-\mathrm{L}-\mathrm{Rh}$ and $1,2,4-\alpha-\mathrm{L}-\mathrm{Rha}$, respectively [18]. The signal at $5.29 \mathrm{ppm}$ is also attributed to $\mathrm{H}-1$ of $1,2-\alpha-\mathrm{L}-\mathrm{Rh}$ [18]. The signal at $2.10 \mathrm{ppm}$ corresponds to the existence of acetyl groups [22]. The existence of $3.82 \mathrm{ppm}$ is the signal of methyl esters connecting to 
carboxyl groups of D-GalA [8]. The abroad peaks between 4.45 and $4.65 \mathrm{ppm}$ indicate the existence of $\mathrm{t}-\beta$-D-Gal and 1,4- $\beta$-D-Gal [23]. The abroad peak at $4.96 \mathrm{ppm}$ is attributed to the H-1 of 1,4- $\alpha$-L-GalA [23].

A

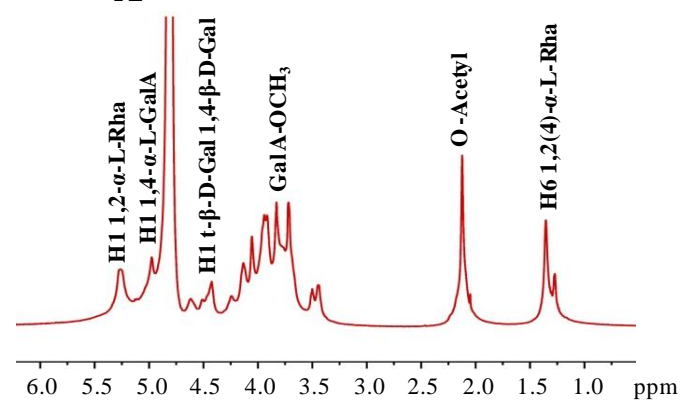

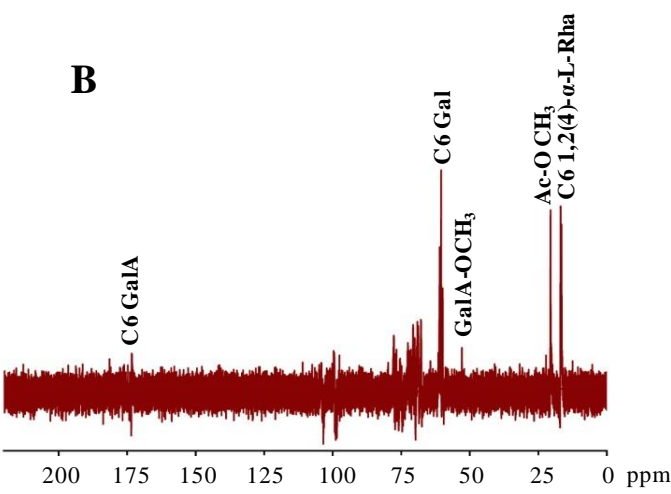

Figure $3 .{ }^{1} \mathrm{H}(\mathbf{A})$ and ${ }^{13} \mathrm{C}(\mathbf{B}) \mathrm{NMR}$ spectra of OPP-D.

The ${ }^{13} \mathrm{C}$ NMR spectra (Figure 3B) of OPP-D showed the C-6 signals for 1,2- $\alpha$-L-Rha and $1,2,4-\alpha-\mathrm{L}-\mathrm{Rha}$ at 16.61 and $16.77 \mathrm{ppm}$ [5]. The presence of $20.63 \mathrm{ppm}$ can be readily attributed to the methyl of acetyl groups [22]. The signals at around $52.48 \mathrm{ppm}$ correspond to the existence of a methyl group esterified carboxyl group of GalA [8,22]. The abroad peaks at around 60.46 ppm are attributed to the C-6 of Gal [10]. The peaks at around $174.1 \mathrm{ppm}$ correspond to the C-6 of un-esterified carbonyl groups of D-GalA [22].

Finally, combined with the constituent monosaccharides and FT-IR spectra of OPP-D as well as the published studies $[10,18]$, these results suggested that the probable structure of OPP-D was -4)- $\alpha$-D-GalAp-(1,2,4)- $\alpha$-L-Rhap-(1- as backbone and 1,4- $\beta$-D-galactan as side chains.

\subsubsection{Molecular Weight and Chain Conformation of OPP-D}

Usually, the bioactivities of polysaccharides are connected with their molecular weight and chain conformation [24]. Thus, the molecular weight, polydispersity $\left(M_{w} / M_{n}\right)$, the radius of gyration $\left(R_{g}\right)$, and chain conformation of OPP-D were investigated. Figure 4A showed that OPP-D was a symmetrical polysaccharide fraction, which suggested that OPP-D was a homogeneous pectic-polysaccharide. Combined with the high content of total polysaccharides and low content of protein, results confirmed that OPP-D was a highly purified polysaccharide. The molecular weight of OPP-D was determined as $2.19 \times 10^{5}( \pm 0.40 \%) \mathrm{Da}$, and the $R_{g}$ of OPP-D was measured at $27.0 \% \pm 3.9 \% \mathrm{~nm}$. The molecular weight of OPP-D was significantly lower than those of polysaccharides extracted by HWE, PWE, and MAE [11], which might be associated with the degradation of polysaccharide by UAE [14,17]. In addition, the polydispersity of OPP-D was $1.76 \% \pm 2.11 \%$, which demonstrated that OPP-D had a relatively broad molecular weight distribution.

The radius of gyration $\left(R_{g}\right)$ is a constant which can characterize the molecular size of the polymer, and $R_{g}$ can be calculated by the equation $R_{g}=\mathrm{kM}_{w}{ }^{\mathrm{v}}[25,26]$. The index $\mathrm{v}$ can be calculated by the linear slope from the configuration diagram constructed by $\log \left(R_{g}\right)$ to $\log \left(M_{w}\right)$, and $v=0.33$ means spherical molecules, $v=0.5-0.6$ means a random string, while $v=1$ means a rigid rod [24]. Figure $4 \mathrm{~B}$ showed the relationship between molecular weights and radius of gyration. As calculated from Figure 4B, the index $\mathrm{v}$ was 0.32 , which indicated that OPP-D existed as a spherical molecule in aqueous solution. 


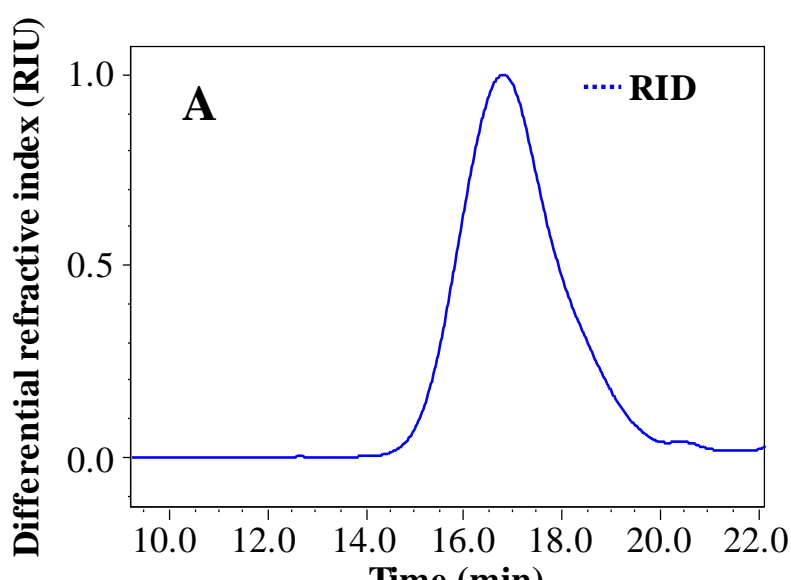

Time (min)
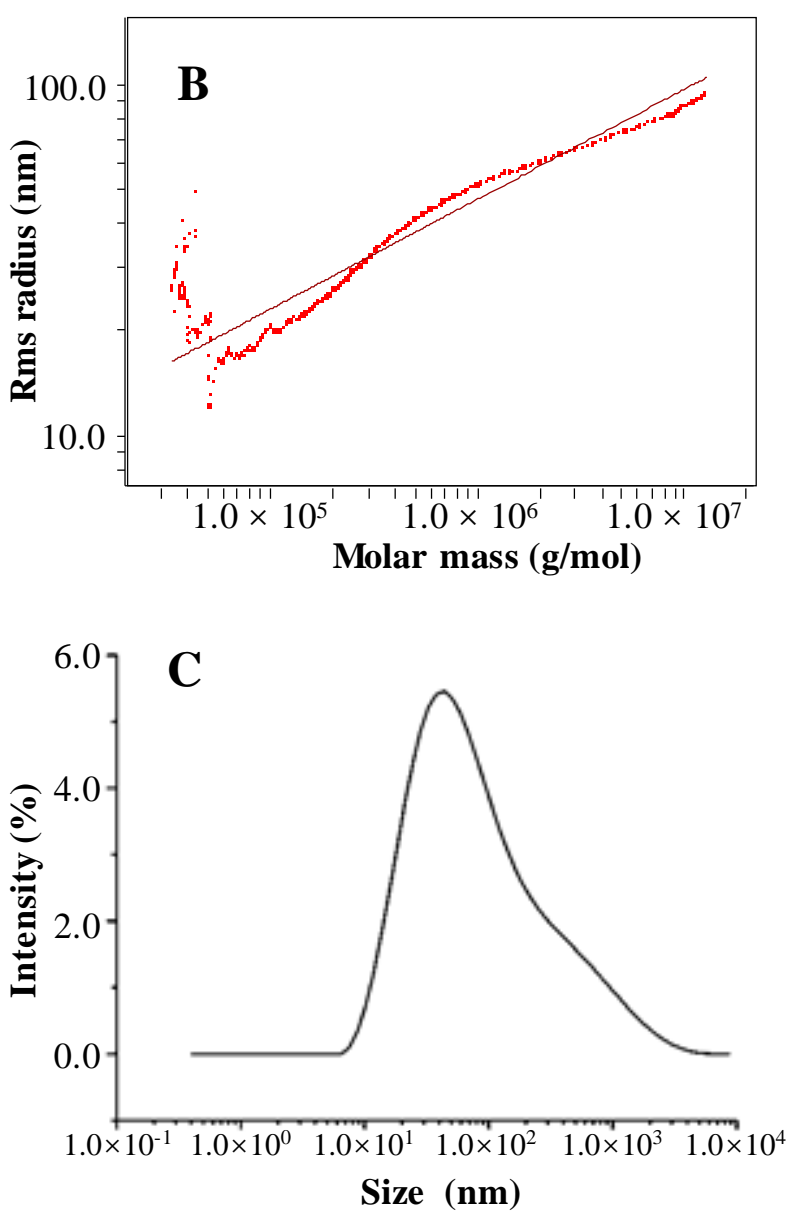

Figure 4. High-performance size-exclusion chromatogram (A), dependence of radius of gyration on molar mass (B), and hydrodynamic radius (C) of OPP-D.

Moreover, the size of polysaccharides in solution can also be determined by dynamic light scattering (DLS), and $R_{h}$ can be calculated by the equation $\mathrm{D}=\mathrm{KT} / 6 \pi \eta \mathrm{r}$ [25]. As shown in Figure $4 \mathrm{C}$, the $R_{h}$ of OPP-D in $0.9 \% \mathrm{NaCl}$ solution was determined as $25.3 \pm 0.1 \mathrm{~nm}$. The structure sensitive parameter $\rho$ can reflect the molecular conformation in solution, and $\rho$ can be calculated by the equation $\rho=R_{g} / R_{h}$ [26]. For a specific polymer solution, the value of $\rho$ represents information of the chain conformation, structure, and polydispersity index of the polymer, and $\rho=0.7-0.8$ means compact sphere, $\rho=1.0-1.1$ means air-core sphere with branching chains, while $\rho=1.5-1.8$ means curly chain 
without rules. The $\rho$ of OPP-D was calculated to be 1.07, which meant that OPP-D existed an air-core sphere with branching chains in aqueous solution [24].

\subsubsection{Apparent Viscosity of OPP-D}

It is well believed that the apparent viscosity of polysaccharide is closely related to molecular characteristics [27]. The apparent viscosities of OPP-D solutions are shown in Figure 5. As shown in Figure 5, the apparent viscosities of OPP-D increased with the increase of concentration, which is similar to the previous study [9]. This property might be associated with the initiation of individual molecular overlapping and increasing the formation of intermolecular connections, resulting in limited alignment and stretching of the polymer chains, and increased the apparent viscosity [28]. In addition, the apparent viscosity of OPP-D decreased with increasing shear rate. The OPP-D solutions exhibited non-Newtonian shear-thinning behaviors at 0.01-50 s $\mathrm{s}^{-1}$, while nearly Newtonian flow behavior at 50-100 s ${ }^{-1}$. For such shear-thinning behavior may mainly be associated with the untangling of the molecular chains in solution [28,29]. Moreover, compared to our previous study, OPP-D exhibited a significantly lower apparent viscosity than OPPs extracted by HWE [9], which mainly due to relatively lower molecular weight and wider polydispersity of OPP-D [30]. All these results suggested that OPP-D possessed typical shear-thinning behavior.

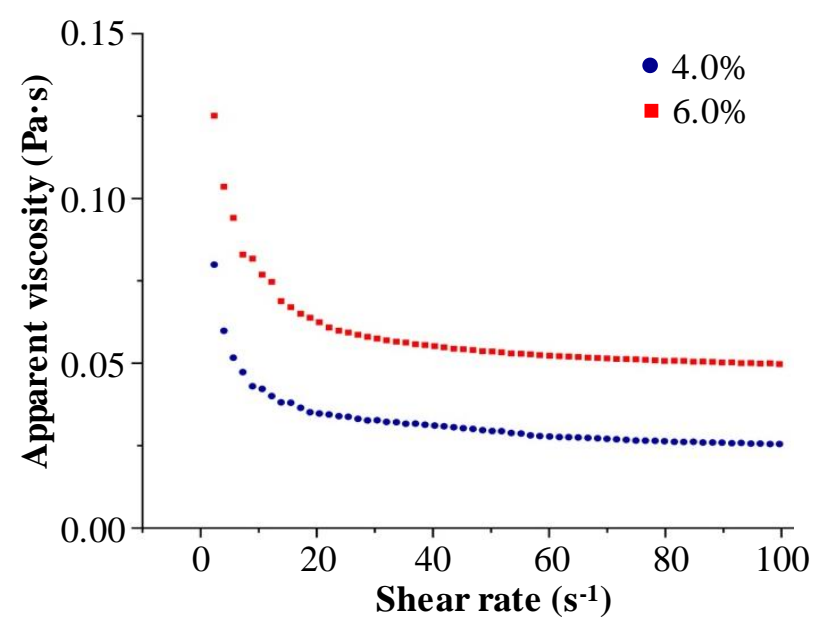

Figure 5. Dependence of apparent viscosity on the shear rate of OPP-D.

\subsection{Antioxidant Activities of OPP-D}

Previously reported studies have already reported that polysaccharides from okra possess remarkable antioxidant activities $[9,11]$. Therefore, the antioxidant activities of OPP-D were measured. The 2,2'-azino-bis (3-ethylbenzthiazoline-6-sulphonic acid) (ABTS) and NO radical scavenging activities of OPP-D are shown in Figure 6. As shown in Figure 6A,B, the ABTS and NO radical scavenging activities of OPP-D demonstrated a dose-dependent manner, respectively, and both reached the highest scavenging activities at $4.50 \mathrm{mg} / \mathrm{mL}$, which indicated that OPP-D exhibited remarkable antioxidant activities. In brief, the $\mathrm{IC}_{50}$ values of $\mathrm{ABTS}$ and $\mathrm{NO}$ radical scavenging activities of OPP-D were evaluated as $2.47 \mathrm{mg} / \mathrm{mL}$ and $0.98 \mathrm{mg} / \mathrm{mL}$, respectively. Additionally, compared to the positive control (vitamin C), OPP-D demonstrated good ABTS and NO radical scavenging activities. Moreover, the antioxidant activities of OPP-D were similar to OPPs extracted by MAE, but relatively higher than those of polysaccharides extracted by HWE and PWE from our previous study [11]. The longer ultrasonic exposure time tends to lower molecular weight and viscosity, shorter chain length, and more hydroxyl groups [31]. The antioxidant activities of natural polysaccharides are associated with their $M_{w}$, uronic acids, and constituent monosaccharides [15,32]. Thus, the relatively high antioxidant activities of OPP-D may be owing to its high content of unmethylated galacturonic acid [9,11]. Results also 
suggested that OPP-D as a natural antioxidant had great potential applications in the food and medical industry.
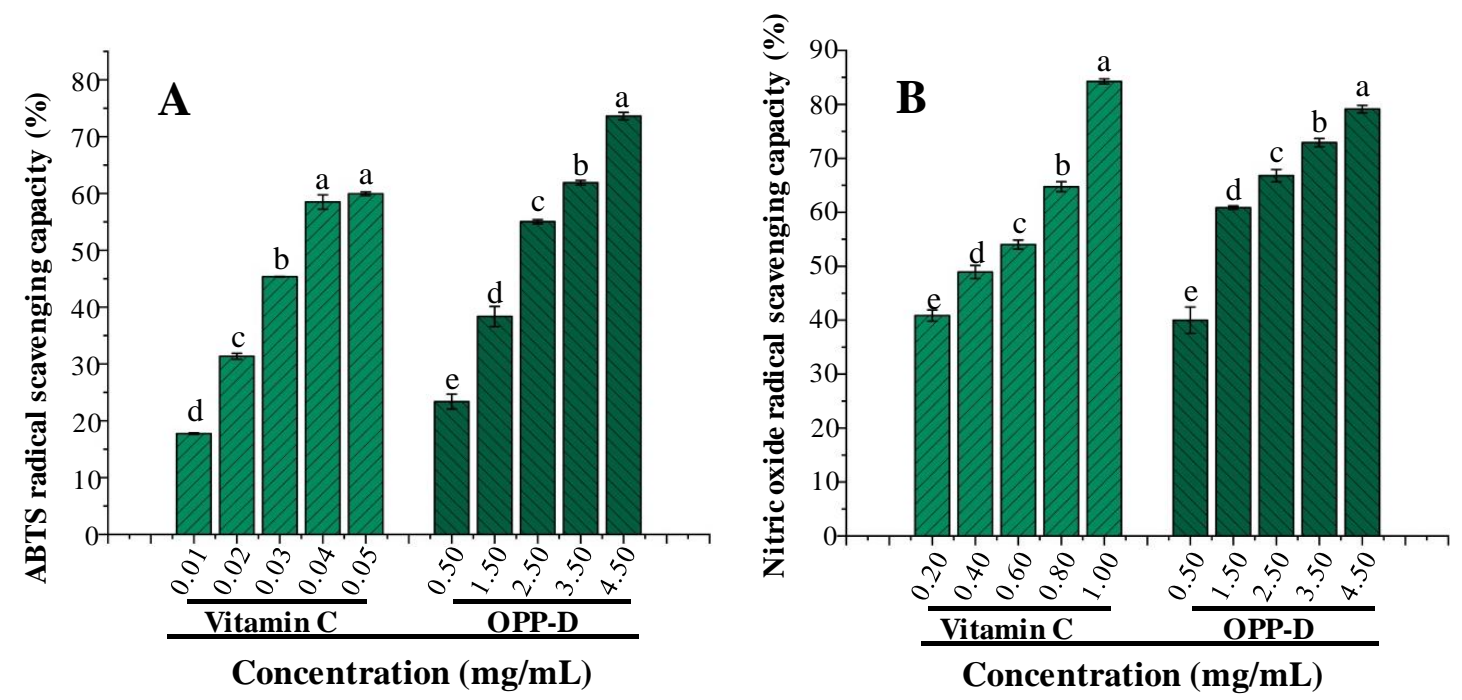

Figure 6. 2,2'-Azino-bis (3-ethylbenzthiazoline-6-sulphonic acid) ABTS radical cation scavenging activity (A) and nitric oxide radical scavenging activity (B) of OPP-D. The error bars are standard deviations. Significant $(p<0.05)$ differences are shown by data bearing different letters $(\mathrm{a}-\mathrm{e})$. Statistical significances were carried out by ANOVA and Duncan's test.

\subsection{In Vitro $\alpha$-amylase and $\alpha$-glucosidase Inhibitory Activities of OPP-D}

The major strategies for counteracting metabolic changes associated with hyperglycemia and type 2 diabetes are the inhibitions of $\alpha$-amylase and $\alpha$-glucosidase [33], and our previous studies have reported that OPPs possessed significant in vitro anti-hyperglycemic activity [9,11]. Therefore, in vitro $\alpha$-amylase and $\alpha$-glucosidase inhibitions of OPP-D were evaluated (Figure 7A,B). Results demonstrated that in vitro $\alpha$-amylase and $\alpha$-glucosidase inhibitions of OPP-D exhibited a dose-dependent manner, and OPP-D showed significant in vitro $\alpha$-amylase and $\alpha$-glucosidase inhibitions. The $\mathrm{IC}_{50}$ values of in vitro $\alpha$-amylase and $\alpha$-glucosidase inhibitions of OPP-D were determined as $223.77 \mu \mathrm{g} / \mathrm{mL}$ and $215.70 \mu \mathrm{g} / \mathrm{mL}$, respectively. Compared with acarbose $\left(\mathrm{IC}_{50}=2020.41 \mu \mathrm{g} / \mathrm{mL}\right)$, the in vitro $\alpha$-glucosidase inhibition of OPP-D was significantly stronger. Furthermore, the in vitro digestive enzyme inhibitions of OPP-D were slightly lower than OPPs extracted by HWE, PWE, and MAE [9,11]. The inhibitions on the digestive enzyme of OPP-D were significantly higher than that of pectic-polysaccharides from Annona squamosa [34] and bitter gourd [32], which might be related to its relatively high $M_{w}$, high DE, and high content of uronic acids [9,20]. Moreover, the highly branched globular conformation of OPP-D in aqueous solution may contribute to available active hydroxyl groups to exert the anti-diabetic activity [35]. Results also suggested that OPP-D as the treatment of type 2 diabetes had great potential applications in the food and medical industry. 

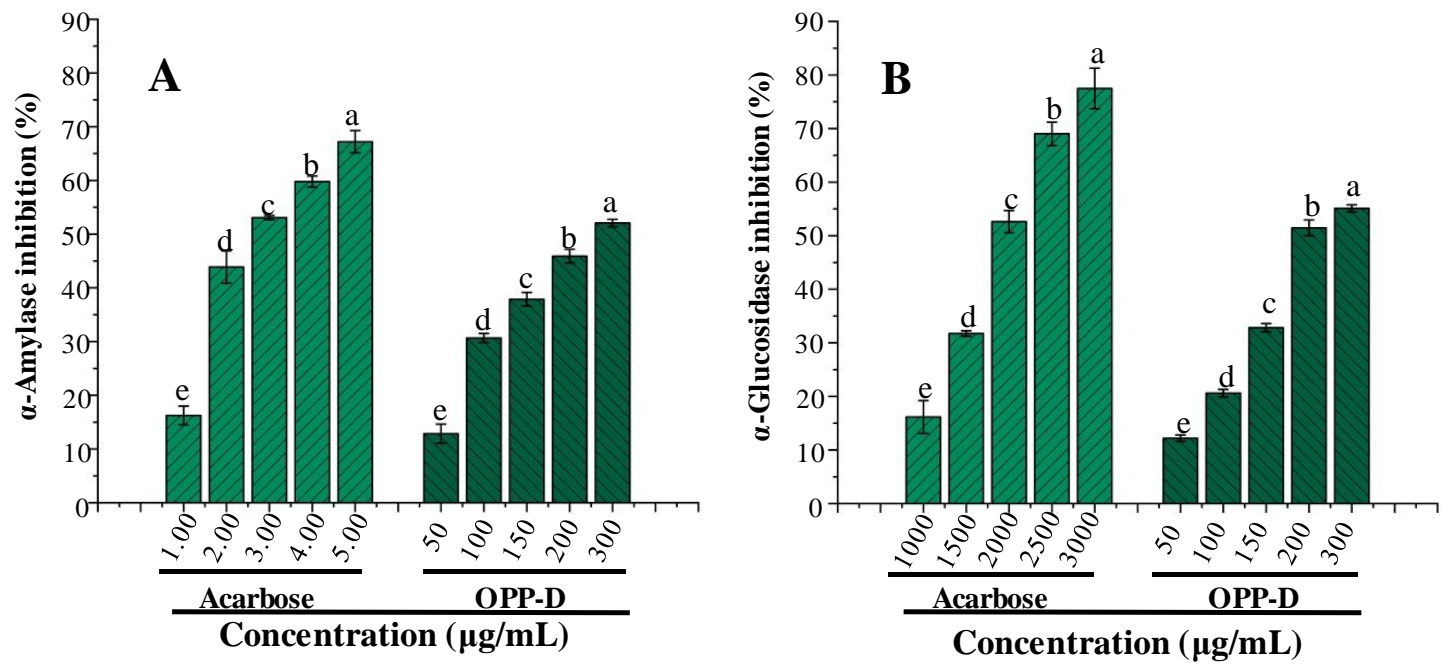

Figure 7. In vitro inhibitory activities on $\alpha$-amylase (A) and $\alpha$-glucosidase (B) of OPP-D. The error bars are standard deviations. Significant $(p<0.05)$ differences are shown by data bearing different letters (a-e). Statistical significances were carried out by ANOVA and Duncan's test.

\subsection{In Vitro Binding Properties of OPP-D}

Excessive absorption of bile acids, cholesterol, and fat may lead to obesity problems, which are related to diabetes cancer and cardiovascular disease [16], and our previous studies have reported that OPPs possess significant in vitro binding properties $[9,11]$. The fat, cholesterol, and bile acid-binding capacities of OPP-D were determined to be $1.47 \pm 0.23 \mathrm{mg} / \mathrm{mg}, 19.34 \pm 1.38 \mathrm{mg} / \mathrm{g}$, and $39.61 \% \pm 0.37 \%$, respectively. Compared with the positive controls, OPP-D showed slightly stronger in vitro binding properties. Furthermore, OPP-D showed lower in vitro binding properties than OPPs extracted by HWE, PWE, and MAE. OPP-D showed similar in vitro binding properties to the $\beta$-glucans from Qingke [16], and higher than that of pectic-polysaccharides bitter gourd [32]. The in vitro binding properties of OPP-D might be related to its DE, $M_{w}$, and molecular weight distributions $[9,20]$. Moreover, such high in vitro binding properties may also be attributed to the highly branched globular conformation of OPP-D and strong hydrogen bonds of carboxylic and hydroxyl groups [36]. Results suggested that OPP-D as functional food ingredients had good potential applications to prevent hypercholesterolemia and hyperlipidemia.

\subsection{In Vitro Prebiotic Activities of OPP-D}

Previous studies have reported that polysaccharides exert their health benefits called prebiotic activities in the human intestine, regulating the production of short-chain fatty acids (SCFAs) [37]. Short-chain fatty acids (SCFAs) are the main end products generated of undigestible carbohydrate fibers by bacterial fermentation, and may contribute to the acidic environment that can inhibit the growth of pathogens in the human gut, thereby altering the intestinal bacterial composition and improving the health of the host [38]. The effects of OPP-D on the growth of three Lactobacilli strains, including L. acidophilus CICC 6089, L. rhamnosus CICC 6133, and L. rhamnosus CICC 6151 were investigated to determine whether OPP-D was a potential substrate to be metabolized by colon microbiota. As shown in Table 1, the number of all tested Lactobacilli strains treated with OPP-D and the total SCFAs were significantly increased, respectively. These meant that OPP-D was not toxic to the assayed probiotics, whereas it was a good substrate for facilitating probiotic growth, and OPP-D could be utilized by probiotic bacteria to sustain survival and metabolic activities. Polysaccharides comprised of galactose may have typically displayed greater prebiotic activity [38,39], and the linkages of polysaccharides are also essential for prebiotic activities, which RG-I may have significant proliferative effects $[37,40]$. Additionally, notable increases in the $\mathrm{OD}_{600}$ were observed which ranged from 0.28 to 0.35 , from 0.33 to 0.40 , and from 0.32 to 0.40 , respectively. Furthermore, similar results could be found in the production 
of SCFAs, and the total SCFAs ranged from 16.17 to $31.32 \mathrm{mM}$, from 10.28 to $20.81 \mathrm{mM}$, and from 54.38 to $90.98 \mathrm{mM}$, respectively. Results showed that all tested Lactobacilli strains exhibited a significantly dose-dependent manner, where a higher content of polysaccharides could have a better proliferative effect [41]. Moreover, the inulin, as for positive control, possessed greater prebiotic activities than OPP-D at the same concentration. Polysaccharides with lower $M_{w}$, better water solubility, and lower viscosity may exert better prebiotic effect [37]. All these results indicated that OPP-D had good potential applications as functional food ingredients for prebiotic activity.

Table 1. Effect of OPP-D on the growth and production of short-chain fatty acids of three probiotics after fermentation for $24 \mathrm{~h}$.

\begin{tabular}{cccc}
\hline Bacteria & Carbon Source & OD $_{600}$ & Total SCFAs (mM) \\
\hline & Control & $0.23 \pm 0.01^{\mathrm{d}}$ & $8.47 \pm 0.98^{\mathrm{d}}$ \\
& 1\% OPP-D & $0.28 \pm 0.02^{\mathrm{cd}}$ & $16.17 \pm 1.53^{\mathrm{d}}$ \\
L. acidophilus CICC 6089 & 2\% OPP-D & $0.31 \pm 0.01^{\mathrm{cd}}$ & $28.46 \pm 2.27^{\mathrm{c}}$ \\
& 3\% OPP-D & $0.35 \pm 0.01^{\mathrm{c}}$ & $31.32 \pm 2.78^{\mathrm{c}}$ \\
& 1\% Inulin & $0.47 \pm 0.03^{\mathrm{b}}$ & $30.34 \pm 1.92^{\mathrm{c}}$ \\
& 2\% Inulin & $0.63 \pm 0.01^{\mathrm{a}}$ & $41.99 \pm 3.25^{\mathrm{b}}$ \\
& 3\% Inulin & $0.65 \pm 0.06^{\mathrm{a}}$ & $51.56 \pm 3.76^{\mathrm{a}}$ \\
\hline & Control & $0.29 \pm 0.01^{\mathrm{c}}$ & $3.51 \pm 0.45^{\mathrm{e}}$ \\
& 1\% OPP-D & $0.33 \pm 0.02^{\mathrm{b}}$ & $10.28 \pm 0.87^{\mathrm{de}}$ \\
& 2\% OPP-D & $0.36 \pm 0.01^{\mathrm{b}}$ & $13.24 \pm 2.16^{\mathrm{cde}}$ \\
& 3\% OPP-D & $0.40 \pm 0.01^{\mathrm{a}}$ & $20.81 \pm 1.85^{\mathrm{bc}}$ \\
& 1\% Inulin & $0.35 \pm 0.01^{\mathrm{b}}$ & $16.94 \pm 2.65^{\mathrm{cd}}$ \\
& 2\% Inulin & $0.40 \pm 0.00^{\mathrm{a}}$ & $28.40 \pm 2.79^{\mathrm{b}}$ \\
& 3\% Inulin & $0.43 \pm 0.00^{\mathrm{a}}$ & $67.04 \pm 6.04^{\mathrm{a}}$ \\
\hline & Control & $0.24 \pm 0.02^{\mathrm{e}}$ & $6.36 \pm 1.17^{\mathrm{c}}$ \\
& 1\% OPP-D & $0.32 \pm 0.01^{\mathrm{d}}$ & $54.38 \pm 4.38^{\mathrm{b}}$ \\
& 2\% OPP-D & $0.33 \pm 0.01^{\mathrm{cd}}$ & $77.64 \pm 6.13^{\mathrm{a}}$ \\
& 3\% OPP-D & $0.40 \pm 0.02^{\mathrm{bc}}$ & $90.98 \pm 10.52^{\mathrm{a}}$ \\
& 1\% Inulin & $0.47 \pm 0.01^{\mathrm{b}}$ & $54.14 \pm 3.56^{\mathrm{b}}$ \\
& 2\% Inulin & $0.55 \pm 0.01^{\mathrm{a}}$ & $89.67 \pm 6.76^{\mathrm{a}}$ \\
& 3\% Inulin & $0.62 \pm 0.05^{\mathrm{a}}$ & $100.89 \pm 8.57^{\mathrm{a}}$ \\
\hline
\end{tabular}

OPP-D, polysaccharide extracted by UAE and purified; SCFAs, short-chain fatty acids; Inulin was used as a positive control in prebiotic activity. Values represent mean \pm standard deviation, and superscripts a-e differ significantly $(p<0.05)$ among OPPs. Statistical significances were carried out by ANOVA and Duncan's test.

\section{Materials and Methods}

\subsection{Material, Chemicals, and Lactobacillus Strains}

Okra fruits were harvested at a commercial orchard in Chengdu, Sichuan, China. The samples were washed, hot-air-dried $\left(75^{\circ} \mathrm{C}\right.$ and $\left.12 \mathrm{~h}\right)$, smashed, screened, and stored at $-20{ }^{\circ} \mathrm{C}$. Acarbose, $\alpha$-amylase (1000 U/mg), $\alpha$-glucosidase (10 U/mg), arabinose (Ara), galactose (Gal), galacturonic acid (GalA), glucose (Glc), glucuronic acid (GlcA), mannose (Man), rhamnose (Rha), xylose (Xyl), 2,2' -azino-bis (3-ethylbenzthiazoline-6-sulphonic acid) (ABTS), 1-phenyl-3-methyl-5-pyrazolone (PMP), and 4-nitrophenyl $\beta$-D-glucopyranoside (pNPG) were all purchased from Sigma-Aldrich (St. Louis, MO, USA). A free cholesterol assay kit and DEAE Cellulose-52 were purchased from Solarbio (Beijing, China). Lactobacillus acidophilus CICC 6089, Lactobacillus rhamnosus CICC 6133, and Lactobacillus rhamnosus CICC 6151 were purchased from China Center of Industrial Culture Collection. All other chemicals and reagents used were of analytical grade.

\subsection{Extraction and Preparation of OPPS}

Extraction and preparation of OPPs were performed by the formerly reported method with minor modifications [11,19]. In brief, $40.0 \mathrm{~mL}$ of phosphate buffer solutions (50 mM, pH 6.0) were used to 
extract OPPs twice by ultrasonic-assisted extraction with an Ultrasonic Processor $(650 \mathrm{~W}, 24 \mathrm{kHz}$, Scientz, Ningbo, China) at room temperature. The ultrasound extraction amplitude and time were set as $75 \%$ and $20 \mathrm{~min}$, respectively. After extraction, the extracts were precipitated with three volumes of $95 \%(v / v)$ ethanol overnight at $4{ }^{\circ} \mathrm{C}$. Subsequently, the precipitations were redissolved, dialyzed for 3 days (Dialysis membrane, molecular weight cut off: $3.5 \mathrm{kDa}$, Solarbio, Beijing, China), freeze-dried, and stored at $-20{ }^{\circ} \mathrm{C}$.

\subsection{Isolation and Purification of OPPS}

OPPs were purified by using a DEAE Cellulose-52 column $(5 \times 50 \mathrm{~cm})$. Briefly, OPPs were dissolved in $40.0 \mathrm{~mL}$ of deionized water at the concentration of $25.0 \mathrm{mg} / \mathrm{mL}$ and filtered through a $0.45 \mu \mathrm{m}$ syringe filter (JinTeng Company, Tianjin, China). The solution was then loaded onto a DEAE Cellulose-52 column and successively eluted by the deionized water and the $0.1 \mathrm{~mol} / \mathrm{L}$ of $\mathrm{NaCl}$ solution at a flow rate of $1.0 \mathrm{~mL} / \mathrm{min}$. Each tube $(5 \mathrm{~mL} /$ tube $)$ was checked at $490 \mathrm{~nm}$ by the phenol-sulfuric acid method. Finally, one purified polysaccharide fraction named OPP-D was obtained (about $0.62 \mathrm{~g}$ ), and the purity of OPP-D was checked by high-performance size-exclusion chromatography.

\subsection{Structural Characterization of OPP-D}

\subsubsection{Chemical Composition Analysis}

The contents of total polysaccharides, total proteins, and total uronic acids of OPP-D were evaluated by the phenol-sulfuric acid assay, Bradford's method, and the $m$-hydroxydiphenyl assay, respectively [11].

\subsubsection{Determination of Constituent Monosaccharides}

Constituent monosaccharides of OPP-D were investigated by HPLC analysis (U3000, Thermo Fisher Scientific, Waltham, MA, USA) based on the previous method [11]. Ara (0.25 M), Gal (0.25 M), GalA (0.5 M), Glc (0.5 M), GlcA (0.5 M), Man (0.5 M), Rha (0.5 M), and Xyl (0.25 M) were mixed and used as a mixture standard solution.

\subsubsection{Fourier Transform Infrared Spectroscopy Analysis}

The Fourier transform infrared (FT-IR) spectroscopy analysis of OPP-D was also investigated by the previous method [11]. The Nicolet iS 10 FT-IR (Thermo Fisher Scientific, Waltham, MA, USA) was used for the determination of the IR spectra of OPP-D in the frequency range of $4000-400 \mathrm{~cm}^{-1}$. The DE of OPP-D was also evaluated from FT-IR spectra on the basis of the previous methods [11,19].

\subsubsection{NMR Analysis}

The OPP-D sample $\left(20 \mathrm{mg}\right.$ ) was dissolved in $0.5 \mathrm{~mL}$ of $\mathrm{D}_{2} \mathrm{O}$ overnight for NMR analysis. 1D NMR spectra, including ${ }^{1} \mathrm{H}$ and ${ }^{13} \mathrm{C}$ analyses, were recorded on a Bruker Ascend $600 \mathrm{MHz}$ spectrometer (Bruker, Rheinstetten, Germany) with a z-gradient probe with proton and carbon frequencies of 600.13 and $150.90 \mathrm{MHz}$, respectively.

\subsubsection{Determination of Molecular Weight and Particle Size}

The purity, absolute molecular weight $\left(M_{w}\right)$, polydispersity $\left(M_{w} / M_{n}\right)$, and radius of gyration $\left(R_{g}\right)$ of OPP-D were determined by high-performance size-exclusion chromatography coupled with multi-angle laser light scattering and refractive index detector (HPSEC-MALLS-RID, Wyatt Technology Co., Santa Barbara, CA, USA) on the basis of the previous method [9].

The hydrodynamic radius $\left(R_{h}\right)$ of OPP-D was measured by using a dynamic light scattering (DLS, Zetasizer Nano, ZEN3600, Malvern Instruments, UK) according to a previous study with some modifications [25]. The sample was dissolved in $0.9 \%$ of $\mathrm{NaCl}$ aqueous solution. The determination 
was carried out at a constant temperature of $25^{\circ} \mathrm{C}$ and at a scattering angle of $90^{\circ}$. The wavelength of the laser beam was set as $633 \mathrm{~nm}$ and the signal acquisition time was $5 \mathrm{~s}$ for 10 times.

\subsubsection{Determination of Apparent Viscosity}

The apparent viscosity of OPP-D was measured by the previous method [9]. In brief, two concentrations $(4.0 \%$ and $6.0 \%, w / v)$ of OPP-D were selected and dissolved in distilled water, respectively. The apparent viscosity was determined by a Discovery Hybrid Rheometer-1 (DHR-1, TA instruments, New Castle DE, USA) equipped with a $40 \mathrm{~mm}$ diameter parallel steel plate with a $1.0 \mathrm{~mm}$ gap. Flow curves of OPP-D were determined at $25^{\circ} \mathrm{C}$ with the shear rate range from 0.01 to $100 \mathrm{~s}^{-1}$.

\subsection{Evaluation of Bioactivities of OPP-D}

\subsubsection{Determination of In Vitro Antioxidant Activities}

Both ABTS and nitric oxide (NO) radical scavenging activities of OPP-D were evaluated by the formerly reported methods [11]. In brief, vitamin $C$ was used as positive control. The ABTS and NO radical scavenging activities of OPP-D were evaluated at five different concentrations, respectively, and on the basis of a logarithmic regression curve, the $\mathrm{IC}_{50}$ values $(\mathrm{mg} / \mathrm{mL})$ of OPP-D could be determined.

\subsubsection{Determination of In Vitro $\alpha$-amylase and $\alpha$-glucosidase Inhibitory Activities}

The inhibitory activities of OPP-D against $\alpha$-amylase and $\alpha$-glucosidase were evaluated by the previously reported methods [11]. The acarbose and distilled water were used as positive and blank controls for both $\alpha$-amylase and $\alpha$-glucosidase inhibitory activities. The $\alpha$-amylase and $\alpha$-glucosidase inhibitory activities of OPP-D were evaluated at five different concentrations, respectively, and on the basis of a logarithmic regression curve, the $\mathrm{IC}_{50}$ values $(\mathrm{mg} / \mathrm{mL})$ of OPP-D could be calculated.

\subsubsection{Determination of In Vitro Binding Properties}

The in vitro binding properties of OPP-D, including fat, cholesterol, and bile acid-binding capacities, were evaluated by the previously reported methods [9,11]. In brief, for the investigation of fat and cholesterol-binding capacities of OPP-D, deionized water, and carboxymethyl cellulose were used as negative and positive controls, respectively. The fat binding capacity of OPP-D was expressed as milligram of binding fat per milligram of OPP-D $(\mathrm{mg} / \mathrm{mg})$. The cholesterol-binding capacity of OPP-D was expressed as milligram of binding cholesterol per gram of OPP-D (mg/g). Moreover, the cholestyramine was used as a positive control for the investigation of bile acid-binding capacity. The bile acid-binding capacity of OPP-D was expressed as a percentage of blank control (\%).

\subsubsection{Determination of In Vitro Prebiotic Activity}

The in vitro prebiotic activity of OPP-D was evaluated by a previous study with minor modifications [37]. Carbohydrate-free MRS broth was used as the basal medium to evaluate the in vitro prebiotic activity. Three Lactobacillus strains, including L. acidophilus CICC 6089, L. rhamnosus CICC 6133, and L. rhamnosus CICC 6151, were selected to determine whether OPP-D was the potential substrate for promotion of the growth of Lactobacillus strains. Inulin and the basal MRS were used as the positive and blank controls, respectively. The samples were prepared as concentrations of 1.0, 2.0, and $3.0 \%(w / v)$, filter-sterilized, and then added into the MRS broth. Each Lactobacillus strain was transferred into MRS broth medium at a concentration of $1 \times 10^{7} \mathrm{CFU} / \mathrm{mL}$. Afterwards, L. acidophilus CICC 6089 and L. rhamnosus CICC 6151 were incubated at $37^{\circ} \mathrm{C}$ for $24 \mathrm{~h}$, and L. rhamnosus CICC 6133 was incubated at $30^{\circ} \mathrm{C}$ for $24 \mathrm{~h}$, respectively. Finally, the optical density values of samples were measured at $600 \mathrm{~nm}$.

SCFAs were determined by gas chromatography according to a previous study with minor modifications [38]. In brief, the fermented broths were centrifuged at $6000 \times g$ for $10 \mathrm{~min}$. Then, $0.4 \mathrm{~mL}$ of supernatants were mixed with $0.4 \mathrm{~mL}$ of internal standard ( $0.05 \mathrm{M}$ of 2-ethylbutyric acid), and filter 
through a $0.22 \mu \mathrm{m}$ membrane filter. The mixed solution was analyzed by Agilent 7890 series GC system (Agilent Technologies, Palo Alto, CA, USA) with an HP-INNOWAX column $(30 \mathrm{~m} \times 0.25 \mathrm{~cm} \times 0.25 \mu \mathrm{m}$, Agilent, USA).

\subsection{Statistical Analysis}

All experiments were conducted in triplicate, and data were expressed in means \pm standard deviations. Statistical significances were carried out by one-way analysis of variance (ANOVA), taking a level of $p<0.05$ as significant to Duncan's multiple range test.

\section{Conclusions}

In this study, the chemical structure, chain conformation, and biological activities of a purified polysaccharide (OPP-D) were investigated. Results showed that OPP-D was a purified pectic-polysaccharide mainly composed of Rha, GalA, and Gal, and the proposed structure of OPP-D was Rha and GalA units as the backbone with galactan side chains. The chain conformation of OPP-D in aqueous solution was determined as an air-core sphere with branching chains for the first time. In addition, OPP-D exhibited remarkable in vitro antioxidant activities, anti-diabetic activity, and binding capacities, as well as prebiotic effect, which might be associated with its $M_{w}, \mathrm{DE}$, content of uronic acids, and highly branched globular conformation. Results suggested that OPP-D had great potential applications to be further explored in the food and medical industries. Based on the favorable effects of OPP-D on prebiotic activities, further studies about the effects of OPP-D on intestinal microorganisms through the in vitro fermentation model and in vivo mice model could be considered.

Author Contributions: D.-T.W. and W.Q. conceived and designed the experiments. X.-R.N. and Y.F. performed the experiments. D.-T.W., W.Q., X.-R.N., Y.F., L.Z. and Q.Z. analyzed the data. Y.F., T.-T.H., Q.J., D.-R.L. and H.C. contributed reagents/materials/analysis tools. X.-R.N. and Y.F. wrote the initial draft. D.-T.W. revised the manuscript. All authors have read and agreed to the published version of the manuscript.

Funding: This work was supported by the National Natural Science Foundation of China (grant number 31901690), the Scientific Research Fund Project of Department of Science and Technology of Sichuan Province (grant number 2018JY0149), and the Scientific Research Foundation of Sichuan Agricultural University (grant number 03120321).

Conflicts of Interest: The authors declare that there are no conflict of interest.

\section{References}

1. Peng, C.H.; Chyau, C.C.; Wang, C.J.; Lin, H.T.; Huang, C.N.; Ker, Y.B. Abelmoschus esculentus fractions potently inhibited the pathogenic targets associated with diabetic renal epithelial to mesenchymal transition. Food Funct. 2016, 7, 728-740. [CrossRef] [PubMed]

2. Xia, F.; Zhong, Y.; Li, M.; Chang, Q.; Liao, Y.; Liu, X.; Pan, R. Antioxidant and anti-fatigue constituents of okra. Nutrients 2015, 7, 8846-8858. [CrossRef] [PubMed]

3. Jiang, N.; Liu, C.; Li, D.; Zhang, Z.; Liu, C.; Wang, D.; Niu, L.; Zhang, M. Evaluation of freeze drying combined with microwave vacuum drying for functional okra snacks: Antioxidant properties, sensory quality, and energy consumption. LWT-Food Sci. Technol. 2017, 82, 216-226. [CrossRef]

4. Wang, K.; Li, M.; Wen, X.; Chen, X.; He, Z.; Ni, Y. Optimization of ultrasound-assisted extraction of okra (Abelmoschus esculentus (L.) Moench) polysaccharides based on response surface methodology and antioxidant activity. Int. J. Biol. Macromol. 2018, 114, 1056-1063. [CrossRef]

5. Zhang, T.; Xiang, J.; Zheng, G.; Yan, R.; Min, X. Preliminary characterization and anti-hyperglycemic activity of a pectic polysaccharide from okra (Abelmoschus esculentus (L.) Moench). J. Funct. Foods 2018, 41, 19-24. [CrossRef]

6. Islam, M.T. Phytochemical information and pharmacological activities of okra (Abelmoschus esculentus): A literature-based review. Phytother. Res. 2019, 33, 72-80. [CrossRef]

7. Chen, Y.; Zhang, J.G.; Sun, H.J.; Wei, Z.J. Pectin from Abelmoschus esculentus: Optimization of extraction and rheological properties. Int. J. Biol. Macromol. 2014, 70, 498-505. [CrossRef]

8. Kpodo, F.M.; Agbenorhevi, J.K.; Alba, K.; Bingham, R.J.; Oduro, I.N.; Morris, G.A.; Kontogiorgos, V. Pectin isolation and characterization from six okra genotypes. Food Hydrocoll. 2017, 72, 323-330. [CrossRef] 
9. Nie, X.R.; Li, H.Y.; Du, G.; Lin, S.; Hu, R.; Li, H.Y.; Zhao, L.; Zhang, Q.; Chen, H.; Wu, D.T.; et al. Structural characteristics, rheological properties, and biological activities of polysaccharides from different cultivars of okra (Abelmoschus esculentus) collected in China. Int. J. Biol. Macromol. 2019, 139, 459-467. [CrossRef]

10. Liu, J.; Zhao, Y.; Wu, Q.; John, A.; Jiang, Y.; Yang, J.; Liu, H.; Yang, B. Structure characterisation of polysaccharides in vegetable "okra" and evaluation of hypoglycemic activity. Food Chem. 2018, 242, 211-216. [CrossRef]

11. Yuan, Q.; Lin, S.; Fu, Y.; Nie, X.R.; Liu, W.; Su, Y.; Han, Q.H.; Zhao, L.; Zhang, Q.; Lin, D.R.; et al. Effects of extraction methods on the physicochemical characteristics and biological activities of polysaccharides from okra (Abelmoschus esculentus). Int. J. Biol. Macromol. 2019, 127, 178-186. [CrossRef]

12. Tao, Y.; Sun, D.-W. Enhancement of food processes by ultrasound: A review. Crit. Rev. Food. Sci. Nutr. 2014, 55, 570-594. [CrossRef]

13. Zhang, D.Y.; Wan, Y.; Xu, J.Y.; Wu, G.H.; Li, L.; Yao, X.H. Ultrasound extraction of polysaccharides from mulberry leaves and their effect on enhancing antioxidant activity. Carbohydr. Polym. 2016, 137, 473-479. [CrossRef]

14. Cheung, Y.C.; Yin, J.; Wu, J.Y. Effect of polysaccharide chain conformation on ultrasonic degradation of curdlan in alkaline solution. Carbohydr. Polym. 2018, 195, 298-302. [CrossRef]

15. Duan, M.; Shang, H.; Chen, S.; Li, R.; Wu, H. Physicochemical properties and activities of comfrey polysaccharides extracted by different techniques. Int. J. Biol. Macromol. 2018, 115, 876-882. [CrossRef]

16. Guo, H.; Lin, S.; Lu, M.; Gong, J.D.B.; Wang, L.; Zhang, Q.; Lin, D.R.; Qin, W.; Wu, D.T. Characterization, in vitro binding properties, and inhibitory activity on pancreatic lipase of beta-glucans from different Qingke (Tibetan hulless barley) cultivars. Int. J. Biol. Macromol. 2018, 120 (Pt B), 2517-2522. [CrossRef]

17. Zhi, Z.; Chen, J.; Li, S.; Wang, W.; Huang, R.; Liu, D.; Ding, T.; Linhardt, R.J.; Chen, S.; Ye, X. Fast preparation of RG-I enriched ultra-low molecular weight pectin by an ultrasound accelerated Fenton process. Sci. Rep. 2017, 7, 1-11. [CrossRef]

18. Thole, C.; Brandt, S.; Ahmed, N.; Hensel, A. Acetylated rhamnogalacturonans from immature fruits of Abelmoschus esculentus inhibit the adhesion of helicobacter pylori to human gastric cells by interaction with outer membrane proteins. Molecules 2015, 20, 16770-16787. [CrossRef]

19. Guo, H.; Yuan, Q.; Fu, Y.; Liu, W.; Su, Y.H.; Liu, H.; Wu, C.Y.; Zhao, L.; Zhang, Q.; Lin, D.R.; et al. Extraction optimization and effects of extraction methods on the chemical structures and antioxidant activities of polysaccharides from snow chrysanthemum (Coreopsis tinctoria). Polymers 2019, 11, 215. [CrossRef]

20. Fu, Y.; Yuan, Q.; Lin, S.; Liu, W.; Du, G.; Zhao, L.; Zhang, Q.; Lin, D.R.; Liu, Y.T.; Qin, W.; et al. Physicochemical characteristics and biological activities of polysaccharides from the leaves of different loquat (Eriobotrya japonica) cultivars. Int. J. Biol. Macromol. 2019, 135, 274-281. [CrossRef]

21. Han, Q.H.; Liu, W.; Li, H.Y.; He, J.L.; Guo, H.; Lin, S.; Zhao, L.; Chen, H.; Liu, Y.W.; Wu, D.T.; et al. Extraction optimization, physicochemical characteristics, and antioxidant activities of polysaccharides from Kiwifruit (Actinidia chinensis Planch.). Molecules 2019, 24, 461. [CrossRef] [PubMed]

22. Alba, K.; Laws, A.P.; Kontogiorgos, V. Isolation and characterization of acetylated LM-pectins extracted from okra pods. Food Hydrocoll. 2015, 43, 726-735. [CrossRef]

23. Sengkhamparn, N.; Verhoef, R.; Schols, H.A.; Sajjaanantakul, T.; Voragen, A.G.J. Characterisation of cell wall polysaccharides from okra (Abelmoschus esculentus (L.) Moench). Carbohydr. Res. 2009, 344, 1824-1832. [CrossRef] [PubMed]

24. Yang, L.; Zhang, L.-M. Chemical structural and chain conformational characterization of some bioactive polysaccharides isolated from natural sources. Carbohydr. Polym. 2009, 76, 349-361. [CrossRef]

25. Liu, W.; Liu, Y.; Zhu, R.; Yu, J.; Lu, W.; Pan, C.; Yao, W.; Gao, X. Structure characterization, chemical and enzymatic degradation, and chain conformation of an acidic polysaccharide from Lycium barbarum $\mathrm{L}$. Carbohydr. Polym. 2016, 147, 114-124. [CrossRef]

26. Wu, D.T.; Lam, S.C.; Cheong, K.L.; Wei, F.; Lin, P.C.; Long, Z.R.; Lv, X.J.; Zhao, J.; Ma, S.C.; Li, S.P. Simultaneous determination of molecular weights and contents of water-soluble polysaccharides and their fractions from Lycium barbarum collected in China. J. Pharm. Biomed. Anal. 2016, 129, 210-218. [CrossRef]

27. Li, L.; Liao, B.Y.; Thakur, K.; Zhang, J.G.; Wei, Z.J. The rheological behavior of polysaccharides sequential extracted from Polygonatum cyrtonema Hua. Int. J. Biol. Macromol. 2018, 109, 761-771. [CrossRef]

28. Benchabane, A.; Bekkour, K. Rheological properties of carboxymethyl cellulose (CMC) solutions. Colloid Polym. Sci. 2008, 286, 1173-1180. [CrossRef] 
29. Jin, Q.; Cai, Z.; Li, X.; Yadav, M.P.; Zhang, H. Comparative viscoelasticity studies: Corn fiber gum versus commercial polysaccharide emulsifiers in bulk and at air/liquid interfaces. Food Hydrocoll. 2017, 64, 85-98. [CrossRef]

30. Zhong, K.; Zhang, Q.; Tong, L.; Liu, L.; Zhou, X.; Zhou, S. Molecular weight degradation and rheological properties of schizophyllan under ultrasonic treatment. Ultrason. Sonochem. 2015, 23, 75-80. [CrossRef]

31. Qiu, J.; Zhang, H.; Wang, Z. Ultrasonic degradation of polysaccharides from Auricularia auricula and the antioxidant activity of their degradation products. LWT-Food Sci. Technol. 2019, 113, 108266. [CrossRef]

32. Yan, J.K.; Wu, L.X.; Qiao, Z.R.; Cai, W.D.; Ma, H. Effect of different drying methods on the product quality and bioactive polysaccharides of bitter gourd (Momordica charantia L.) slices. Food Chem. 2019, 271, 588-596. [CrossRef]

33. Podsedek, A.; Majewska, I.; Redzynia, M.; Sosnowska, D.; Koziolkiewicz, M. In vitro inhibitory effect on digestive enzymes and antioxidant potential of commonly consumed fruits. J. Agric. Food Chem. 2014, 62, 4610-4617. [CrossRef]

34. Ren, Y.Y.; Zhu, Z.Y.; Sun, H.Q.; Chen, L.J. Structural characterization and inhibition on $\alpha$-glucosidase activity of acidic polysaccharide from Annona squamosa. Carbohydr. Polym. 2017, 174, 1-12. [CrossRef]

35. Liu, H.; He, P.; He, L.; Li, Q.; Cheng, J.; Wang, Y.; Yang, G.; Yang, B. Structure characterization and hypoglycemic activity of an arabinogalactan from Phyllostachys heterocycla bamboo shoot shell. Carbohydr. Polym. 2018, 201, 189-200. [CrossRef]

36. Yang, L.; Fu, S.; Zhu, X.; Zhang, L.; Yang, Y.; Yang, X.; Liu, H. Hyperbranched Acidic Polysaccharide from Green Tea. Biomacromolecules 2010, 11, 3395-3405. [CrossRef]

37. Huang, F.; Liu, H.; Zhang, R.; Dong, L.; Liu, L.; Ma, Y.; Jia, X.; Wang, G.; Zhang, M. Physicochemical properties and prebiotic activities of polysaccharides from longan pulp based on different extraction techniques. Carbohydr. Polym. 2019, 206, 344-351. [CrossRef]

38. Chen, G.; Chen, X.; Yang, B.; Yu, Q.; Wei, X.; Ding, Y.; Kan, J. New insight into bamboo shoot (Chimonobambusa quadrangularis) polysaccharides: Impact of extraction processes on its prebiotic activity. Food Hydrocoll. 2019, 95, 367-377. [CrossRef]

39. Mueller, M.; Cavarkapa, A.; Unger, F.M.; Viernstein, H.; Praznik, W. Prebiotic potential of neutral oligo- and polysaccharides from seed mucilage of Hyptis suaveolens. Food Chem. 2017, 221, 508-514. [CrossRef]

40. Khodaei, N.; Fernandez, B.; Fliss, I.; Karboune, S. Digestibility and prebiotic properties of potato rhamnogalacturonan I polysaccharide and its galactose-rich oligosaccharides/oligomers. Carbohydr. Polym. 2016, 136, 1074-1084. [CrossRef]

41. Wang, X.; Huang, M.; Yang, F.; Sun, H.; Zhou, X.; Guo, Y.; Wang, X.; Zhang, M. Rapeseed polysaccharides as prebiotics on growth and acidifying activity of probiotics in vitro. Carbohydr. Polym. 2015, 125, 232-240. [CrossRef]

Sample Availability: Samples of the raw material of okra and the purified okra polysaccharides are available from the authors.

(C) 2020 by the authors. Licensee MDPI, Basel, Switzerland. This article is an open access article distributed under the terms and conditions of the Creative Commons Attribution (CC BY) license (http://creativecommons.org/licenses/by/4.0/). 